\title{
Relationship of serum IL-6, C-reactive protein, erythrocyte sedimentation rate, and knee skin temperature after total knee arthroplasty: a prospective study
}

\author{
Sittisak Honsawek • Benjamad Deepaisarnsakul • Aree Tanavalee • \\ Manoon Sakdinakiattikoon • Srihatach Ngarmukos • Kanok Preativatanyou • \\ Piyanuch Bumrungpanichthaworn
}

Received: 10 January 2010 /Revised: 22 January 2010 /Accepted: 25 January 2010 /Published online: 21 February 2010

(C) Springer-Verlag 2010

\begin{abstract}
Knee osteoarthritis is a common cause of severe pain and functional limitation. Total knee arthroplasty is an effective procedure to relieve pain, restore knee function, and improve quality of life for patients with end stage knee arthritis. The aim of this study was to investigate the inflammatory process in patients with primary knee osteoarthritis before surgery and in subsequent periods following total knee arthroplasty. A prospective study of 49 patients undergoing primary total knee replacements was conducted. The patients were evaluated by monitoring serum interleukin-
\end{abstract}

Presented at the sixth SICOT/SIROT (combined SICOT/RCOST 2009) annual meeting, Pattaya, Thailand, October 29-November 1, 2009 and presented in part at the Orthopaedic Research Society 56th Annual Meeting, New Orleans, Louisiana, USA, March 6-9, 2010.

S. Honsawek · B. Deepaisarnsakul $\cdot$ K. Preativatanyou •

P. Bumrungpanichthaworn

Department of Biochemistry, Faculty of Medicine,

Chulalongkorn University,

Bangkok 10330, Thailand

S. Honsawek $\cdot$ A. Tanavalee $\cdot$ S. Ngarmukos

Department of Orthopaedics, Faculty of Medicine,

Chulalongkorn University,

Bangkok 10330, Thailand

\section{Sakdinakiattikoon}

Department of Orthopaedic Surgery,

Bangkok Metropolitan Administration General Hospital,

Bangkok 10100, Thailand

\section{S. Honsawek $(\square)$}

Department of Biochemistry and Orthopaedics, Faculty of Medicine, Chulalongkorn University,

King Chulalongkorn Memorial Hospital,

Rama IV Road, Patumwan,

Bangkok 10330, Thailand

e-mail: Sittisak.H@chula.ac.th
6 (IL-6), C-reactive protein (CRP), erythrocyte sedimentation rate (ESR), knee skin temperature, and clinical status. Measurements were carried out preoperatively and postoperatively on day one and at two, six, 14, and 26 weeks during follow-up review in the knee clinic. The serum IL-6 and CRP were elevated on the first postoperative day but fell to preoperative values at two weeks postoperatively. Both returned to within the normal range by six weeks postoperatively. In addition, the postoperative ESR showed a slow rise with a peak two weeks after surgery and returned to the preoperative level at 26 weeks postoperatively. The difference in skin temperature between operated and contralateral knees had a mean value of $+4.5^{\circ} \mathrm{C}$ at two weeks. The mean value decreased to $+3.5^{\circ} \mathrm{C}$ at six weeks, $+2.5^{\circ} \mathrm{C}$ at 14 weeks, and $+1.0^{\circ} \mathrm{C}$ at 26 weeks. The difference in skin temperature decreased gradually and eventually there was no statistically significant difference at 26 weeks after surgery. A sustained elevation in serum IL-6, CRP, ESR, and skin temperature must raise the concern of early complication and may suggest the development of postoperative complication such as haematoma and/or infection.

\section{Introduction}

Osteoarthritis (OA) is one of the most common degenerative joint diseases that progressively results in loss of joint function and can lead to substantial morbidity and disability in the elderly [1]. Knee osteoarthritis is the leading cause of severe pain and functional limitation. Total knee arthroplasty (TKA) is an effective procedure to relieve pain, restore knee function, and improve quality of life for patients with end stage knee arthritis.

C-reactive protein (CRP) and erythrocyte sedimentation rate (ESR) are very useful routine laboratory screening tests 
for diagnosis of potential infection. ESR is a nonspecific haematological test routinely used as an indirect parameter of increased acute phase reactants. CRP is a major acute phase reactant that is produced by the liver in response to inflammation, infection, malignancy, and tissue damage $[2,3]$. The synthesis of CRP is rapidly upregulated, primarily in hepatocytes, under the control of the proinflammatory cytokines.

Interleukin-6 (IL-6) is produced substantially by monocytes and macrophages after antigen activation, even though other cells (such as fibroblasts, endothelial cells, and T-lymphocytes) may also synthesise it [4]. Surgery may instantly mediate IL-6 release by these cells, or IL-6 release may be activated by other locally released cytokines. Synthesis of IL-6 in fibroblasts can be upregulated by both IL-1 and tumour necrosis factor (TNF)- $\alpha$ [5]. However, levels of TNF- $\alpha$, IL-1 $\beta$, and IL- 8 are often very low for detection. TNF- $\alpha$ and IL- $1 \beta$ are most difficult to measure in the systemic circulation even in severely infected patients [6]. Furthermore, IL-6 is principally responsible for activating the hepatic synthesis of CRP, which has been considered the inflammatory biomarker of choice in orthopaedic surgery $[7,8]$.

The objective of this study was to investigate the inflammatory process in patients with primary knee osteoarthritis before surgery and in subsequent periods following total knee replacement. This study was designed to evaluate the serial changes of serum IL-6, CRP, ESR, knee skin temperature, and clinical status after uncomplicated TKA. In addition, we investigated whether there were differences in temporal values of IL-6, CRP, and ESR and further determined the relationships of these parameters with knee skin temperature post TKA.

\section{Patients and methods}

\section{Patients}

This study was approved by the Institutional Review Board on Human Research of the Faculty of Medicine, Chulalongkorn University and was conducted in compliance with the guidelines of the Declaration of Helsinki. Written informed consent was obtained from the patients prior to their participation in the study.

Forty-nine patients, aged 50-78 years, diagnosed with primary knee osteoarthritis (40 females and nine males; mean age $67.8 \pm 1.1$ years) were recruited in this prospective study over a 26 -week period. We excluded any patients with inflammatory arthritis, a systemic inflammatory or autoimmune disorder, or a history of any type of cancer or chronic illness. All patients underwent total knee arthroplasty at our institute from September 2007 to August 2008 and were operated upon by one surgeon (AT) using the standard surgical technique. The routine anaesthesia method was spinal block and when spinal anaesthesia was ineffective, general anaesthesia was administered. In each case the standard parapatellar approach was used with a tourniquet. Femoral, tibial and patellar components were NexGen (Zimmer, Warsaw, Indiana, USA) using polymethylmethacrylate cement Palacos R containing gentamycin (Heraeus Medical $\mathrm{GmbH}$, Germany). A wound drain with vacuum suction was used and was removed on the first day after surgery. All patients were mobilised on the second operative day.

The skin temperature of both knees was evaluated postoperatively at two, six, 14, and 26 weeks during follow-up review in the knee clinic. The temperature was determined using digital pocket surface thermometers at four different locations on the anterior aspect of the knee (superomedial, superolateral, inferomedial, and inferolateral border of the patella). The mean of the two readings was taken as the final temperature. A similar measurement was also carried out on the contralateral knee, which served as the control. Assessment taken at midday was selected as the time for taking readings for the rest of the study. For each patient, the same thermometer was used throughout the course of the study.

\section{Laboratory methods}

Venous blood samples were obtained before surgery, and on the first postoperative day and at two, six, 14, and 26-weeks follow-up. The blood samples were centrifuged to remove cells and debris, and stored at $-80^{\circ} \mathrm{C}$ until analysis. Creactive protein was measured using the latex particle enhanced nephelometric immune assay on the BN ProSpec analyser (Dade Behring, Germany) and was expressed in milligrams per decilitre $(\mathrm{mg} / \mathrm{dl})$. Erythrocyte sedimentation rate was determined using the Westergren method. The concentrations of IL- 6 in serum were performed by commercially available human IL-6 enzyme-linked immunosorbent assay (ELISA) MAX sets (Biolegend, San Diego, CA) according to the manufacturer's instructions. Concisely, 96-well microtiter plates were precoated with monoclonal antibody supplied in the kit, and a blocking solution was added to eliminate nonspecific antigen binding. After washing, the wells were incubated with either serum or known concentration standards of recombinant human IL-6 at room temperature. After plate washing, a biotin-labelled detection antibody was added. Following incubation and washing, an avidinhorseradish peroxidase enzyme and color substrate was applied for the detection of binding. Lastly, the reaction was stopped with the stop solution, and then absorbance was measured at $450 \mathrm{~nm}$ using an automated microplate reader. Recombinant human IL-6 was used to develop a standard curve. 
Table 1 Comparison of serum IL-6, CRP, and ESR levels before and after surgery. The data are expressed as mean $\pm \operatorname{standard~deviation(SD)}$

\begin{tabular}{|c|c|c|c|c|c|c|}
\hline \multirow[t]{2}{*}{ Parameter } & \multirow[t]{2}{*}{ Preoperative } & \multicolumn{5}{|l|}{ Postoperative } \\
\hline & & 24 hours & Two weeks & Six weeks & 14 weeks & 26 weeks \\
\hline IL-6 (pg/ml) & $57.0 \pm 21.2$ & $184.5 \pm 53.5^{*}$ & $66.3 \pm 23.1$ & $55.3 \pm 22.4$ & $55.7+28.4$ & $55.9+20.8$ \\
\hline $\operatorname{ESR}(\mathrm{mm} / \mathrm{hr})$ & $26.5 \pm 17.6$ & $47.5 \pm 27.0$ & $58.0 \pm 28.3$ & $42.0 \pm 27.2$ & $40.6 \pm 20.5$ & $39.5 \pm 26.1$ \\
\hline CRP (mg/dl) & $3.1 \pm 2.6$ & $85.0 \pm 21.7^{*}$ & $6.9 \pm 4.8$ & $4.4 \pm 2.5$ & $3.1 \pm 2.3$ & $3.1 \pm 2.1$ \\
\hline
\end{tabular}

IL-6 interleukin-6, ESR erythrocyte sedimentation rate, CRP C-reactive protein

* $P<0.0001$

\section{Statistical analysis}

Statistical analysis was performed using the Statistical Package for Social Sciences software, version 16.0 for Windows (SPSS Inc., Chicago, IL, USA). All values were expressed as mean \pm standard deviation (SD). Comparisons between groups were employed using Student's $t$-test and Mann-Whitney $U$-test. A $P$-value $<0.05$ was considered to indicate statistical significance.

\section{Results}

There were no complications within the study period or on further follow-up. Patients who developed complications following knee replacement were excluded from the study. The values of serum IL-6, CRP, and ESR are shown in Table 1. Serum IL-6 concentration elevated rapidly and peaked 24 hours postoperatively at maximum levels $(184.5 \pm$ $53.5 \mathrm{pg} / \mathrm{ml}$ ). In addition, the CRP levels increased and reached maximum levels $(85.0 \pm 21.7 \mathrm{mg} / \mathrm{dl})$ within the first postoperative day in all patients. Both serum IL-6 and CRP fell to preoperative values at two weeks and returned to

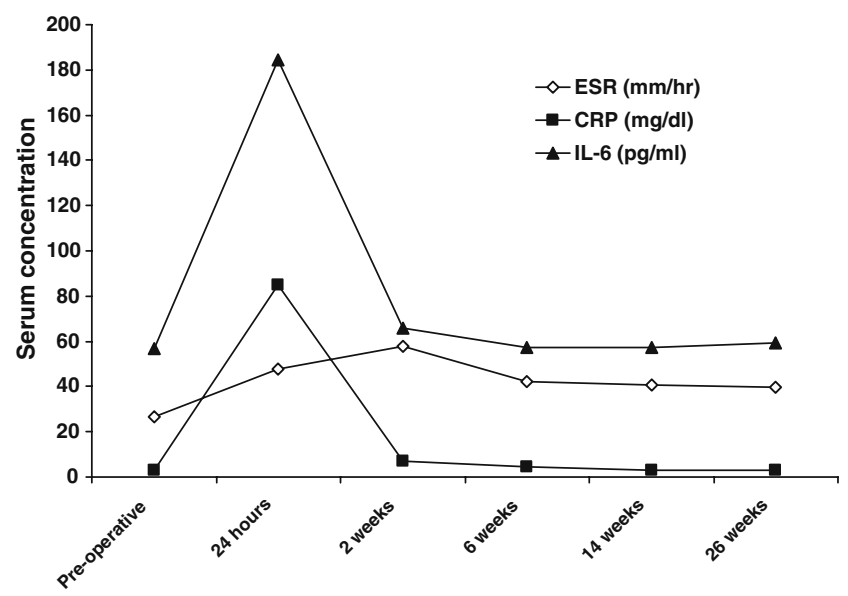

Fig. 1 Comparison between mean serum interleukin-6 (IL-6), Creactive protein (CRP), and erythrocyte sedimentation rate (ESR) in osteoarthritis (OA) patients following total knee arthroplasty (TKA) within the normal range by six weeks postoperatively. Comparison of these two inflammatory markers demonstrates a statistically significant difference during the first 24 postoperative hours $(P<0.0001)$.

In comparison, the postoperative ESR showed a slow rise with a peak two weeks after surgery $(58.0 \pm 28.3 \mathrm{~mm} /$ $\mathrm{hr}$ ) and returned to the preoperative level at 26 weeks postoperatively. The temporal patterns of mean levels of IL6, CRP, and ESR before and after surgery were demonstrated in Fig. 1. The difference in skin temperature between operated and contralateral knees had a mean value of $+4.5^{\circ} \mathrm{C}$ at two weeks (Fig. 2). The mean value decreased to $+3.5^{\circ} \mathrm{C}$ at six weeks, $+2.5^{\circ} \mathrm{C}$ at 14 weeks, and $+1.0^{\circ} \mathrm{C}$ at 26 weeks. The difference in skin temperature decreased gradually and eventually there was no statistically significant difference at 26 weeks after surgery $(P>0.05)$ (Fig. 2).

\section{Discussion}

Infection following joint arthroplasty is a major concern for surgeons and other medical practitioners. Nonspecific clinical presentation makes it difficult to distinguish from

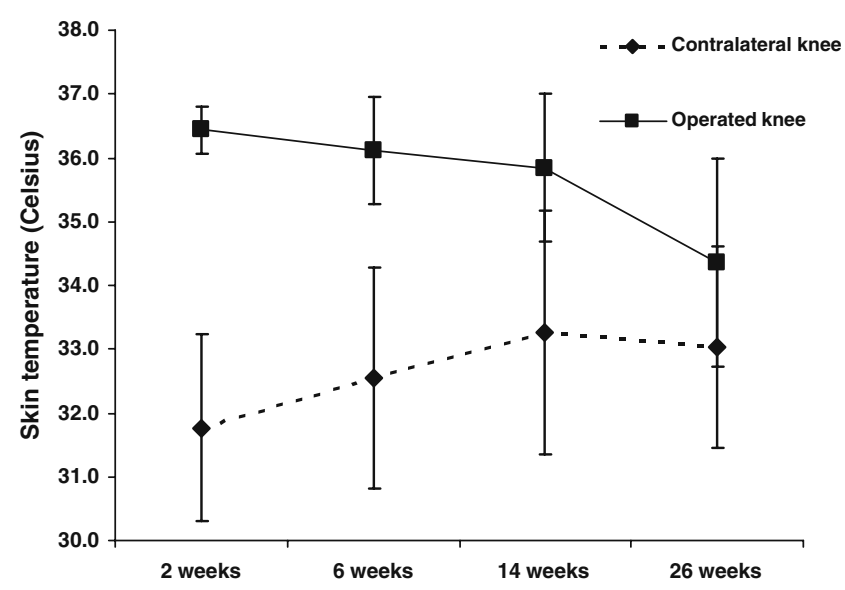

Fig. 2 Mean skin temperature of both knees in osteoarthritis patients following total knee arthroplasty (TKA). The data are expressed as mean \pm standard deviation (SD) 
those indicating infection. Objective data are needed to exclude the possibility of infection despite suspicious clinical symptoms and signs. CRP and ESR have been extensively used to examine the presence of infection following surgery. IL-6 appears to be a major endogenous protein mediator of fever and the acute phase response after surgery [5]. IL-6 also plays a crucial role in the induction and regulation of the acute phase protein response, particularly that of CRP synthesis by hepatocytes [9]. Elevated CRP, ESR, and skin temperature can raise concern that infection may be present following TKA [10]. It is imperative therefore to investigate the temporal patterns of IL-6, CRP, ESR, and knee skin temperature after uncomplicated TKA.

Our results have demonstrated that there is a significant rise in IL-6 and CRP. In comparison, ESR differs in the temporal pattern of postoperative level after TKA. ESR had slower temporal changes and lower amplitude changes. These findings are in accordance with those of previous studies reporting the postoperative levels of IL-6, CRP, and ESR after total joint replacement [11, 12]. Serum IL-6 and CRP correlate with a high degree of inflammatory activity with a more rapid increase and a faster return to normal values than ESR, suggesting that IL-6 and/or CRP measurements could be more favourable than ESR measurements in determining the presence of infection during the early postoperative period. Moreover, previous studies have indicated that IL- 6 may be a better indicator of postoperative inflammatory response [13-15]. IL-6 levels reach the maximum level in the first six to 12 hours following surgery and fall back to their baseline range by 48 to 72 hours postoperatively $[13,16,17]$. CRP levels peak two to three days after surgery and return to normal values approximately three weeks postoperatively [18-20].

This study has also shown that the skin temperature of the operated knee is significantly raised following surgery and gradually decreased by 26 weeks postoperatively. This temperature difference is presumably attributed to the postoperative inflammatory response in the operated knee. In agreement with our findings, Mehra et al. have demonstrated that skin temperature of the operated knee increases in the early postoperative period but returns to normal at 18 weeks postoperatively [21]. Haidar et al. have also shown a decrease in the mean difference between the operated and contralateral knee over time [22].

Significant postoperative elevations in IL-6, CRP, ESR, and knee skin temperature should be a normal response to surgical trauma and not an indication of complications. Measurements of these parameters, including at least one preoperative measurement, are required when the clinical significance of the postoperative values of these parameters is indicated. If postoperative values of these parameters continue to be elevated for several days with respect to the preoperative value, or even show a tendency to rise, further investigation for this must be carefully evaluated.

It should be noted, however, that we are aware of some inherent limitations. Firstly, we did not collect blood specimens during the early postoperative period when CRP and ESR are elevated. This made it difficult to determine precisely when peak CRP and ESR was achieved. No blood sampling was collected between the first and 14th postoperative days, which made it impossible to tell the exact time when CRP and/ or ESR reach the maximum level. Secondly, our study was based on a relatively small number of enrolled patients. A further investigation with a larger number of subjects would be needed to make a strong conclusion. Finally, the results derived from our study cannot necessarily be extrapolated in men because of female predominance of subjects with knee OA.

In conclusion, this study demonstrates the temporal patterns of serum IL-6, CRP, ESR, and knee skin temperature in osteoarthritis patients undergoing TKA. The use of serum IL-6 in conjunction with CRP and ESR was suggested to verify infection following surgery. A sustained elevation in serum IL-6, CRP, ESR, and skin temperature must raise the concern of early complication and may suggest the development of postoperative complication such as haematoma and/or infection.

Acknowledgements This investigation was supported by the Ratchadapiseksompotch Fund, Faculty of Medicine, Chulalongkorn University, Thailand Research Fund, and the National Research Council of Thailand. We are grateful to Chulalongkorn Medical Research Center (ChulaMRC) for kindly providing facilities.

\section{References}

1. Felson DT, Zhang Y, Hannan MT, Naimark A, Weissman BN, Aliabadi P (1995) The incidence and natural history of knee osteoarthritis in the elderly. Arthritis Rheum 38:1500-1505

2. Pepys MB (1981) C-reactive protein fifty years on. Lancet $1: 653-657$

3. Kushner I, Gewurz H, Benson MD (1981) C-reactive protein and the acute phase response. J Lab Clin Med 97:739-749

4. Kishimoto T (1989) The biology of interleukin-6. Blood 74:1-10

5. Walther Z, May LT, Sehgal PB (1988) Transcriptional regulation of the interferon-beta 2/B cell differentiation factor BSF-2/ hepatocyte-stimulating factor gene in human fibroblasts by other cytokines. J Immunol 140:974-977

6. Damas P, Reuter A, Gysen P, Demonty J, Lamy M, Franchimont P (1989) Tumor necrosis factor and interleukin-1 serum levels during severe sepsis in humans. Crit Care Med 17:975-978

7. Larsson S, Thelander U, Friberg S (1992) C-reactive protein (CRP) levels after elective orthopedic surgery. Clin Orthop Relat Res 275:237-242

8. Niskanen RO, Korkala O, Pammo H (1996) Serum C-reactive protein levels after total hip and knee arthroplasty. J Bone Joint Surg Br 78:431-433

9. Heinrich PC, Castell JV, Andus T (1990) Interleukin-6 and the acute phase response. Biochem J 265:621-636 
10. Hanssen AD, Rand JA (1999) Evaluation and treatment of infection at the site of a total hip or knee arthroplasty. Instr Course Lect 48:111-122

11. Minetto MA, Oprandi G, Saba L, Mussino S, Aprato A, Massè A, Angeli A, Gallinaro P (2006) Serum interleukin-6 response to elective total hip replacement surgery. Int Orthop 30:172-176

12. Park KK, Kim TK, Chang CB, Yoon SW, Park KU (2008) Normative temporal values of CRP and ESR in unilateral and staged bilateral TKA. Clin Orthop Relat Res 466:179-188

13. Wirtz DC, Heller KD, Miltner O, Zilkens KW, Wolff JM (2000) Interleukin-6: a potential inflammatory marker after total joint replacement. Int Orthop 24:194-196

14. White J, Kelly M, Dunsmuir R (1998) C-reactive protein level after total hip and total knee replacement. J Bone Joint Surg Br 80:909-911

15. Niskanen RO, Korkala O, Pammo H (1996) Serum C-reactive protein levels after total hip and knee arthroplasty. J Bone Joint Surg Br 78:431-433

16. Baigrie RJ, Lamont PM, Kwiatkowski D, Dallman MJ, Morris PJ (1992) Systemic cytokine response after major surgery. Br J Surg 79:757-760
17. Kragsbjerg P, Holmberg H, Vikerfors T (1995) Serum concentrations of interleukin-6, tumour necrosis factor-alpha, and $\mathrm{C}$ reactive protein in patients undergoing major operations. Eur $\mathrm{J}$ Surg 161:17-22

18. Moreschini O, Greggi G, Giordano MC, Nocente M, Margheritini F (2001) Postoperative physiopathological analysis of inflammatory parameters in patients undergoing hip or knee arthroplasty. Int J Tissue React 23:151-154

19. Bilgen O, Atici T, Durak K, Karaeminoğullari BMS (2001) Creactive protein values and erythrocyte sedimentation rates after total hip and total knee arthroplasty. J Int Med Res 29:7-12

20. Larsson S, Thelander U, Friberg S (1992) C-reactive protein (CRP) levels after elective orthopedic surgery. Clin Orthop Relat Res 275:237-242

21. Mehra A, Langkamer VG, Day A, Harris S, Spencer RF (2005) C reactive protein and skin temperature post total knee replacement. Knee 12:297-300

22. Haidar SG, Charity RM, Bassi RS, Nicolai P, Singh BK (2006) Knee skin temperature following uncomplicated total knee replacement. Knee 13:422-426 